\title{
Grupo Tutorial em Saúde Bucal: a experiência em atenção primária no Centro de Saúde do Itapoã-DF
}

Oral Health Tutorial Group: an experience in Primary
Health Care at the Itapoz Basic Health Unit-DF Programa de Educación Tutorial en Salud Bucal: la experiencia en Atención Primaria en el Centro de Salud de Itapoã-DF

\author{
Tiago Araújo Coelho de Souza \\ Doutor em Saúde Pública \\ Departamento de Odontologia \\ Universidade de Brasília \\ Maria do Carmo Toledo Ramos \\ Secretaria de Estado de Saúde do Distrito Federal \\ Juliana Fiuza Franco \\ Regina Cardoso de Moura \\ Marina Meirelles Bogalho Moita \\ Lorena Gonçalves Vilela \\ Gratia Patricia Lucatelli Nunes \\ Marcelle Cristina Simioni Chupel \\ Clarissa Borges Barbosa \\ Larissa Gavião Junqueira \\ Renata Porto Stypulkowski \\ Renato Costa Vieira da Silva \\ Bolsita(s) PET-Saúde \\ Departamento de Odontologia \\ Universidade de Brasília
}

RESUMO: Este artigo apresenta o relato de experiência do Programa de Educação Tutorial em Odontologia da Universidade de Brasília, durante o período de março a dezembro de 2010. O cenário de prática das atividades de campo é a Regional Administrativa do Itapoã, no Distrito Federal, e a atuação do grupo tutorial acontece junto às Equipes de Saúde da Família por meio de rotinas educativo-preventivas em saúde bucal durante as visitas domiciliares, atividades clínicas e desenvolvimento de pesquisas. Alunos e preceptores estão sendo capazes de reconhecer os determinantes sociais do processo saúdedoença, analisar os indicadores de saúde bucal da população, aprimorar práticas educativas utilizadas na Estratégia Saúde da Família e promover saúde no âmbito da comunidade. As principais dificuldades encontradas dizem respeito a limitação de espaço físico e de recursos capazes de atender a demanda do grupo tutorial e das famílias assistidas. Esta experiência tutorial junto à Estratégia Saúde da Família possibilita um aprendizado que vai muito mais além do que a tradição de ensino bio-tecnicista da odontologia, bem como permite aos discentes de odontologia da UnB 'vivenciar uma experiência real' e refletir sobre os conhecimentos teóricos obtidos nos 'bancos da universidade'. 
Palavras-chave: saúde bucal; odontologia; atenção primária

ABSTRACT: This paper presents an experience report of the Tutorial Program in Dentistry from the University of Brasilia, during the period from March to December, 2010. The scenario of practice is the Itapoã an Administrative Area of the Distrito Federal - and the activities of the tutorial group take place in partnership with the Family Health Team through a preventive educational oral health routine during home visits, as well as clinical activities and development of research. Students and preceptors are being able to recognize the social determinants of the health-disease process, analyze oral health indicators of the population, improve educational practices and promote health within the community. The main barriers of the tutorial group are the lack of physical space and dental equipment capable of meeting the demand of the tutorial group and assisted families. This tutorial experience within the Family Health Strategy provides a learning experience that goes far beyond the tradition of teaching bio-technicalities of dentistry, as well as allows dental students real to live through a real field experience and reflect on the theoretical knowledge obtained from the 'university seats'.

Keywords: oral health; dentistry; primary health care

RESUMEN: Este documento presenta el relato de la experiencia del Programa de Educación Tutorial en Odontología de la Universidad de Brasilia, durante el período de Marzo a Diciembre de 2010. El escenário de práctica de las actividades de campo es el Sector Regional Administrativo de Itapoã, en el Distrito Federal, donde el trabajo del grupo tutorial acompaña el trabajo de los Equipos de Salud de la Família a través de la educación preventiva de rutina en la salud bucal de la población, mejorando las prácticas educativas utilizadas en la estrategia de salud familiar $y$ promoviendo la salud en la comunidad. Las principales dificultades se refieren a la limitación del espacio físico y el equipo dental capaces de satisfacer la demanda del grupo tutorial y de las famílias atendidas. Esta actividad tutorial con la Estrategia de Salud de la Família ofrece una experiencia de aprendizaje que vá más allá de la tradición de la enseñanza de técnicas bio-odontológicas, así como permite a los estudiantes de odontología de la UnB "pasar una verdadera experiencia" $y$ reflexionar sobre el conocimiento teórico obtenido en "los bancos de datos de la universidad".

Palabras-clave: salud bucal; odontología; atención primária de salud

\section{INTRODUÇÃO}

O Programa de Educação pelo Trabalho para a Saúde (PET-Saúde) instituído a partir do Programa de Educação Tutorial (PET) do Ministério da Educação é uma estratégia de fortalecimento da Atenção Básica fomentada pelo Programa Nacional de Reorientação da Formação Profissional em Saúde, o PRÓSAÚDE, em implementação no país desde $2005^{1-3}$. O PET-Saúde consiste na formação de grupos de aprendizagem tutorial compostos por um tutor docente, preceptores (profissionais do serviço) e discentes, que recebem incentivo financeiro na forma de bolsas, para participarem em áreas estratégicas do Sistema Único de Saúde (SUS) e pautarem-se nos princípios do mesmo $^{3-5}$. É premissa do PET-Saúde que os estudantes de graduação conheçam o trabalho na esfera da saúde pública e vivenciem novas experiências, bem como que os profissionais do serviço sejam qualificados/capacitados por meio desta experiência. O PET-Saúde visa estimular a interação entre estudantes, docentes, profissionais e a população, além de visualizar nas necessidades do serviço uma fonte de produção de conhecimento e pesquisa por meio das Instituições de Ensino Superior ${ }^{3-5}$.

Dentro da política de distribuição dos 
campos de estágio da Secretaria de Estado da Saúde do Distrito Federal (SES-DF) para os cursos da área de saúde existentes em Brasília, a Regional de Saúde do Paranoá/ Itapoã tem sido designada, desde 2007, como área prioritária de atuação da Universidade de Brasília $(\mathrm{UnB})^{6}$. O PET-Saúde da UnB se concentra, portanto, nessa região, objetivando contribuir para que a Estratégia Saúde da Família (ESF) seja levada a êxito, através da integração entre a comunidade, os cursos de graduação e as Equipes da ESF localizadas na Regional de Saúde do Paranoá/Itapoã.

Em março de 2010, foi criado de forma pioneira, um grupo tutorial composto de discentes e docentes de graduação de Odontologia da Faculdade de Ciências da Saúde da UnB que além dos objetivos gerais supracitados, visa produzir conhecimento por meio de projetos de pesquisa, a saber: a) capacitação em saúde bucal dos Agentes Comunitários de Saúde (ACS); b) discussão da divisão do trabalho em saúde bucal na ESF do pós-lei 11.889/2008 que regulamenta o exercício das profissões de Técnico em Saúde Bucal (TSB) e de Auxiliar em Saúde Bucal $(\mathrm{ASB})^{7}$; c) monitoramento e avaliação das restaurações de ionômero de vidro; d) realização de inquérito epidemiológico em saúde bucal nos moldes do SB Brasil 2010\%; e e) compreensão das representações sociais acerca dos hábitos de autocuidado e da atenção em saúde bucal da população atendida pela ESF no Itapoã.

As atividades do grupo tutorial de odontologia da UnB contemplam o tripé ensino-pesquisa-extensão e são materializadas por meio de práticas inovadoras de atenção e experiências pedagógicas que contribuem para a reorientação da formação e implementação das Diretrizes Curriculares Nacionais dos cursos de graduação da área da saúde ${ }^{9-12}$. Portanto, o objetivo deste artigo é relatar as atividades desenvolvidas pelo grupo tutorial de odontologia da UnB, bem como discutir assuntos pertinentes à odontologia, na área do ensino em saúde bucal coletiva.

\section{Caracterização do cenário de prática e do} grupo tutorial

O grupo tutorial PET-Odontologia da UnB atua junto `a Regional Administrativa (RA) do Itapoã, que atualmente possui cerca de 100 mil habitantes. Esta Regional tem sua origem na década de 90 a partir de invasões irregulares, em uma região a cerca de $30 \mathrm{Km}$ do Plano Piloto, pertencente à RA de Sobradinho, porém bem próxima da RA do Paranoá. Em 2001, o número de moradores vindos de outros estados e também de cidades vizinhas aumentou com a expectativa de regularização da área e este crecimento populacional foi essencial para a criação em 2003 da Subadministração do Itapoã, vinculada agora à administração regional do Paranoá, por meio da aprovação do Projeto de Lei ${ }^{\circ}$ 698/03 $3^{13}$.

O Centro de Saúde (CS) do Itapoã foi inaugurado em abril de 2009 e está localizado na Quadra 378, Área Especial 1 sob número do Cadastro Nacional de Estabelecimento de Saúde (CNES) 6.268.269. Esse CS serve como base de apoio para o PET-Odontologia da UnB, onde são realizados neste estabelecimento, bem como em suas imediações atendimentos clínicos, atividades educativo-preventivas e pesquisas científicas.

No CS do Itapoã realizam-se ações de saúde voltadas tanto para a livre demanda como para 
a Estratégia Saúde da Família. Nesta unidade básica existem cinco equipes de saúde da família, sendo que apenas três delas possuem equipes de saúde bucal, todas na modalidade $\mathrm{I}^{15}$. O CS ainda conta com a presença do Núcleo de Apoio à Saúde da Família (NASF) o que leva a uma ampliação das ações de saúde e contribui para a integralidade da atenção ao indivíduo ${ }^{16}$.

Anteriormente à inserção da ESF, o atendimento clínico odontológico no Centro de Saúde do Itapoã funcionava por meio de lista de espera. Porém, a lista de espera se tornou tão extensa que aqueles casos onde apenas uma intervenção mínima seria necessária, com a demora, já necessitavam de uma abordagem mais complexa ou invasiva, como Endodontia ou Exodontia. No CS realiza-se também o Programa Cárie Zero, destinado a prestar acompanhamento odontológico a gestantes e crianças de até seis anos de idade.

O grupo PET-Odontologia da UnB é composto por 10 alunos bolsistas, 1 aluna voluntária, 1 tutor e 5 preceptores. Dos alunos, têm-se representantes de vários períodos do curso de odontologia da UnB. Em relação à preceptoria, têm-se 4 dentistas e 1 enfermeira, que possuem experiência tanto na Estratégia Saúde da Família como na livre demanda.

\section{Relato de experiência do grupo tutorial de odontologia da UnB}

A ESF tem como objetivo principal promover uma mudança em como é realizada a atenção à saúde, reorganizando a prática assistencial de modo a causar maior impacto social por englobar medidas preventivas e promocionais que se propõe a restabelecer a saúde integral ${ }^{14}$; também se encarrega de incentivar a mobilização do indivíduo, de modo que esse possa assumir corresponsabilidade pela manutenção de sua saúde. O processo de implantação da ESF modifica a dinâmica de oferta e demanda dos serviços prestados à população, pois cada vez mais as práticas realizadas pelos profissionais de saúde estão se tornando importantes para assegurar a humanização, resolutividade e qualidade dos serviços prestados na rede do SUS.

Toda ação que venha a ser desenvolvida necessita ser planejada antes de aplicada, o que não foi diferente com as atividades desse grupo tutorial. Após a seleção dos alunos, foram realizadas reuniões de planejamento das ações e reconhecimento do ambiente. As preceptoras, servidoras da SES-DF, explicaram acerca do funcionamento da unidade de saúde, bem como apresentaram a rotina da clínica de Odontologia. Posteriormente, os formulários utilizados no serviço, as fichas clínicas, os prontuários das famílias e o funcionamento da referência e contra-referência foram apresentados. Finalmente, um roteiro das atividades a serem realizadas foi acordado entre as partes.

Observou-se que como a ESF era recente na região, a comunidade não estava inteiramente acostumada com o sistema de visitas domiciliares pelos ACS e profissionais de saúde, isso acontecia porque a procura pelos serviços sempre era feita pelo próprio paciente, motivado principalmente por quadros de dor. Os profissionais da equipe odontológica também não estavam completamente confortáveis com a rotina de visitas, pois afirmavam que o processo de formação deles fora centrado em uma vertente clínica, que focava de maneira hegemônica os conceitos da livre demanda, 
do caráter privado, do individualismo e do curativismo $^{17,18}$. Visitas domiciliares são importantes no currículo das Universidades, já que ampliam os conhecimentos, humanizam a atenção e envolvem de forma real o futuro profissional e a comunidade, facilitando o estabelecimento de relações de vínculo que melhorarão o entendimento das partes ${ }^{19,20}$.

As visitas domiciliares do grupo tutorial PET-Odontologia da UnB sempre foram acompanhadas pelos ACS's e aconteciam por meio de uma rotina preventivo-promocional que era também utilizada para capacitar em serviço os próprios agentes comunitários. Nestas visitas realizava-se uma apresentação inicial sobre os objetivos do projeto, esclarecimento quanto ao vínculo dos alunos da UnB com o CS, deixando a critério da família participar ou não do programa. Para as famílias que aceitavam participar, a visita seguia com uma sessão de perguntas a respeito da saúde bucal dos familiares, instrução de higiene oral com auxílio de macromodelos e espelhos, e distribuição de kits de higiene bucal a cada membro da família.

Ainda durante a visita, era realizada a evidenciação de placa nos moradores do domicílio com consequente marcação do Índice de Higiene Oral Simplificado em uma ficha denominada "Boletim de Visitas Domiciliares"21; as visitas seguintes eram realizadas de acordo com a necessidade de cada família para acompanhamento e marcação de consulta.

Os familiares eram agendados para uma primeira consulta odontológica e após o exame clínico, caso nenhuma necessidade clínica fosse constatada, o paciente recebia a notificação na ficha odontológica de Tratamento Completo
(TC) na Atenção Básica. Caso se constatasse necessidade clínica não compatível com as realizadas no $\mathrm{CS}$, o paciente era encaminhado para o atendimento especializado, sendo posteriormente acompanhado por meio de visitas regulares para verificação se o tratamento foi realizado e recebendo Tratamento Completo na especialidade encaminhada (ex. TC Periodontia, TC Exodontia). Caso fosse constatado necessidade de atendimento clínico referente a atenção primária, o paciente iniciava o tratamento no mesmo instante no CS do Itapoã.

Para melhor atender a demanda acumulada da população do Itapoã, optou-se em utilizar a técnica do Tratamento Restaurador Atraumático (TRA). O TRA é uma técnica simples e de baixo custo que segue uma filosofia de promoção da saúde, sendo desenvolvida para evitar que extrações desnecessárias sejam realizadas. Segundo alguns autores ${ }^{22-24}$ o baixo custo desta técnica, o restabelecimento da função e o desempenho clínico satisfatório tornam o TRA uma alternativa viável aos tratamentos convencionais, principalmente, quando utilizada em saúde pública.

A implementação do uso do TRA no CS do Itapoã se mostrou de grande valia para os discentes, uma vez que permitiu que os mesmos fossem expostos `a técnica com maior instensidade, bem como viabilizou a criação de um fórum de discussão privilegiado sobre o TRA e seu impacto no perfil epidemiológico da população. Do ponto de vista da comunidade, a utilização do TRA permitiu dar maior vazão 'a demanda acumulada existente. No entanto, ficou claro para o grupo tutorial a carência de estudos e instrumentos que possibilitem aplicar um método uniforme e objetivo para avaliar 
a qualidade e longevidade das restaurações realizadas por meio desta técnica.

A existência da ESF nos municípios brasileiros depende de uma escolha política, e muitas vezes essa Estratégia é implantada sem planejamento adequado, visando somente o recurso financeiro oferecido pelo Ministério da Saúde. O mesmo acontece com a implementação das equipes de saúde bucal (modalidade I ou II), que carece de um extenso planejamento para que a assistência atenda plenamente a demanda. A presença do TSB no serviço torna o atendimento além de eficaz, eficiente, pois diminui gastos e agiliza o atendimento da demanda acumulada ${ }^{25}$. O que, no entanto, acontece com frequência, é que o rendimento das equipes não é levado em conta nesse processo, assim, a presença do TSB muitas vezes não é o caminho escolhido; e quando é, esse profissional acaba atuando como um 'ASB de luxo', apesar de sua formação e da lei 11.889/2008. Em verdade, este cenário também está presente no CS do Itapoã, e apesar de alguns profissionais serem formados como TSB, estão registrados e consequentemente atuando como ASB.

Outro ponto de interesse do grupo tutorial PET-Odontologia da UnB foram os inquéritos epidemiológicos $^{26,27}$ aos moldes do SB Brasil 2003 e $2010^{28,31}$. Estas duas pesquisas nacionais em saúde bucal representam a incorporação da vigilância à saúde na Política Nacional de Saúde Bucal, bem como a valorização da epidemiologia como um importante recurso nas ações de planejamento, monitoramento e avaliação dos agravos e determinantes do processo saúde-doença. Conhecendo a importância destes estudos, o grupo tutorial se propôs a realizar um inquérito em saúde bucal - segundo os moldes do SB Brasil 2003 e 2010 - em uma amostra de conveniência da população atendida pelo PET-Odontologia. Esta atividade possibilitaria a capacitação dos alunos quanto ao uso de instrumentos de vigilância e permitiria a discussão acerca do perfil epidemiológico encontrado.

No que tange as pesquisas SB Brasil 2003 e 2010, diversos autores discutem sobre planejamento, coleta de dados, amostra, significância dos resultados e sua extrapolação ou não para toda a população brasileira ${ }^{28-32}$. Sendo assim, estes estudos serviram de base para a fundamentação teórica da linha de pesquisa voltada a conhecer o perfil de saúde bucal da população assistida por este grupo tutorial.

No início da aplicação do inquérito, um questionamento foi levantado: o preenchimento da ficha de exame deveria ser realizado em domicílio ou no consultório dentário do CS do Itapoã? Percebeu-se, durante a prática, que o preenchimento em consultório era mais efetivo, pois dispunhamos de melhor iluminação para a observação, secagem do sítio a ser examinado e facilidade na execução da sondagem periodontal. Adicionalmente, a pouca experiência dos alunos fazia com que os exames realizados em ambiente domiciliar levassem um tempo demasiado. Também foi observado que examinar apenas os pacientes nas idades índices tornaria o processo de aprendizagem mais limitado; portanto, a ficha de exame aos moldes do SB Brasil 2010 foi sendo preenchida independentemente da idade, e, incorporado 'a rotina clínica dos alunos. No momento, o grupo está em fase de análise do banco de dados.

As maiores dificuldades encontradas para 
a realização do inquérito aos moldes do $\mathrm{SB}$ Brasil foram: o excesso de variáveis a serem preenchidas pelos alunos-examinadores, gerando demora e dúvidas quando da execução dos exames; a falta de um processo de calibração mais minuncioso; e a limitação de instrumentais necessários.

Por fim, a linha de pesquisa intitulada 'Representações Sociais' tem como objetivo principal a produção de conhecimento sobre o processo de apropriação da população acerca dos hábitos de autocuidado e da atenção em saúde bucal na ESF. Este estudo está sendo realizado por meio de entrevistas em profundidade (técnica do grupo focal) que visam analisar as representações sociais e as características socioeconômicas dos sujeitos pesquisados. Ao final do estudo, têm-se como proposta a elaboração de um instrumento que sirva como veículo para propagação e valorização do conhecimento em saúde bucal da população do Itapoã. No momento, o grupo está na fase de coleta de dados.

As representações se manifestam como elementos cognitivos, mas não se restringem a isso, já que são compartilhadas e contribuem para uma realidade comum. Em verdade, as Representações Sociais podem ser entendidas como interpretações da realidade subjetivamente construídas e mediadas por categorias históricas, culturais, dinâmicas e flexíveis, ou seja, não são o acúmulo de conhecimentos estagnados pelo tempo, ao contrário, são capazes de se reformular de acordo com o avanço da ciência e o desenvolvimento do senso-comum ${ }^{33-36}$.

No contexto da prevenção e educação em saúde a tradição biomédica se mostra ineficaz na maioria das vezes, pois o paciente não consegue compreender e assimilar o conhecimento científico veiculado pelo profissional de saúde de maneira uníssona. Logo, para haver uma relação harmônica e efetiva entre profissional de saúde e sujeito, é importante que o primeiro entenda a realidade sócio-cultural do segundo, ou seja, conheça e valorize as representações sociais que cercam este sujeito, e consiga assim se fazer entender da forma mais adequada e garanta o comprometimento e respeito do paciente.

\section{CONCLUSÃO}

O grupo tutorial PET-Odontologia da UnB oportuniza aos discentes integralizar os conhecimentos obtidos na universidade a um contexto social, bem como possibilita o desenvolvimento de atividades técnicocientíficas e preventivo-promocionais em um 'cenário vivo'. As atividades realizadas são fundamentais para o desenvolvimento de novas práticas de atenção e experiências pedagógicas, concorrendo para a plena integração ensinoserviço-comunidade e para o fortalecimento da atenção básica de acordo com os princípios e necessidades do SUS.

No que tange às preceptoras, foi possível identificar a intensificação de canais de interlocução com a comunidade, adicionalmente à interlocução com os discentes. Esta experiência tutorial propiciou também uma maior qualificação do trabalho da equipe de saúde bucal junto `a ESF e a atualização profissional por meio das pesquisas em desenvolvimento.

Mas nem sempre o sol brilha. Como este grupo foi pioneiro, as atividades praticadas nem sempre encontravam suporte estrutural, sendo que em alguns momentos após as devidas 
avaliações, foram necessárias reformulações das ações realizadas. Há ainda muito a ser melhorado, como a necessidade de espaço físico e de equipamentos capazes de atender plenamente as famílias, busca de alguma alternativa eficiente para divulgação das atividades e dos resultados obtidos, tornar mais racional o planejamento e, por fim, permitir o controle social do programa por meio de algum instrumento que venha a ser discutido com a comunidade, os discentes e preceptores.

A experiência do grupo tutorial em odontologia da UnB propicia um 'ensaio' da capacidade de desenvolver os conhecimentos adquiridos em sala de aula para a vivência da profissão em um ambiente real e, muitas vezes, distante dos ambientes idealizados nos 'bancos universitários'.

\section{AGRADECIMENTOS}

Agradecimentos às preceptoras, Dra. Fernanda Oliveira Veríssimo e Dra. Sandra Aguiar de Oliveira pelo relato da experiência anterior ao início das atividades PETOdontologia; à Coordenadora de Odontologia da RA do Paranoá, Dra. Jaqueline Rodrigues Lourenço que possibilitou a doação de kits de higiene bucal aos familiares; à preceptora, Dra. Cristiane Peres dos Santos que através dela aprendemos sobre o funcionamento do CS; e por fim a aluna voluntária Mariana Graça Lira.

\section{AGÊNCIA DE FOMENTO}

Este trabalho teve ajuda financeira, sob a forma de bolsas, do programa PET-Saúde do Ministério da Saúde.

\section{REFERÊNCIAS}

1. Brasil. Ministério da Saúde. PET-Saúde, 2011. Disponível em: http://portal.saude.gov. br/portal/saude/profissional/visualizar_texto. cfm?idtxt=35306. Acesso em: 19 set. 2011.
2. Brasil. Ministério da Saúde. Pró-Saúde, 2011. Disponível em: http://portal.saude.gov. br/portal/sgtes. Acesso em: 19 set. 2011.

3. Haddad AE, Campos FE, Fruet de Freitas MSB, Brenelli SL, Passarella TM, Ribeiro TCV. Programa de Educação pelo Trabalho para a Saúde-PET-SAÚDE. Cadernos ABEM. 2009; 5(1):6-12.

4. Brasil. Ministério da Saúde. Objetivos e Resultados, 2011. Disponível em: http:// portal.saude.gov.br/portal/saude/profissional/ visualizar texto.cfm?idtxt=32566. Acesso em: 19 set. 2011.

5. Brasil. Ministério da Saúde. Ministério da Educação. Portaria Interministerial $\mathrm{n}^{\mathrm{o}}$ 1.802, de 26 de agosto de 2008. Institui o Programa de Educação pelo Trabalhador para a SaúdePET-Saúde. Diário Oficial da União. Brasília, 27 ago. 2008; Seção 1, p.27.

6. Brasil Ministério da Saúde. Universidade de Brasília. Secretaria de Estado da Saúde do Distrito Federal. PET-SAÚDE 2010. Edital N ${ }^{\circ}$ 18/2009. Seleção para o programa de educação pelo trabalho para a saúde, 2010.

7. Brasil. Lei $\mathrm{n}^{\mathrm{o}}$ 11.889. Regulamenta o exercício das profissões de Técnico em Saúde Bucal - TSB e de Auxiliar em Saúde Bucal ASB. 24 de dezembro de 2008.

8. Projeto SBBrasil 2010 - Pesquisa Nacional de Saúde Bucal. Manual da Equipe de Campo. Disponível em: http://www.sbbrasil2010.org Acesso em: 19 jan. 2011.

9. Assega ML, Lopes Júnior LC, Santos EV, Antoniassi RS, Padula MGC, Pirolo MS. A Interdisciplinaridade vivenciada no PETSaúde. Rev. Ciência \& Saúde. 2010; 3(1):2933. 
10. Brasil. Ministério da Saúde. O SUS de A a Z : garantindo saúde nos municípios. Ministério da Saúde, Conselho Nacional das Secretarias Municipais de Saúde. $3^{\mathrm{a}}$ ed. Brasília: Ministério da Saúde, 2009.

11. Brasil. Ministério da Saúde. Secretaria de Políticas de Saúde. Projeto Promoção da Saúde. As Cartas da Promoção da Saúde. Ministério da Saúde, Secretaria de Políticas de Saúde, Projeto Promoção da Saúde. $1^{\mathrm{a}}$ ed. Brasília: Ministério da Saúde, 2002.

12. Brasil. Ministério da Saúde. Secretaria de Vigilância em Saúde. Secretaria de Atenção à Saúde. Política Nacional de Promoção da Saúde. $3^{\mathrm{a}}$ ed. Brasília: Ministério da Saúde, 2010 .

13. Governo do Distrito Federal. Portal do Cidadão. Administração do Itapoã. Tudo Sobre o Itapoã. Conheça o ItapoãRAXXVIII. Disponível em: http://www.itapoa. df.gov.br/. Acesso em: 12 ago. 2011.

14. Brasil. Ministério da Saúde. Departamento de Atenção Básica. Atenção Básica e a Saúde da Família. Disponível em: http://dab.saude. gov.br/atencaobasica.php. Acesso em: 21 fev. 2011.

15. Brasil. Ministério da Saúde. Departamento de Atenção Básica. Saúde Bucal. Disponível em: http://dab.saude.gov.br/CNSB. Acesso em: 01 abr. 2011.

16. Governo do Distrito Federal. Secretaria de Estado de Saúde. Subsecretaria de Atenção à Saúde. Diretoria de Atenção Primária a Saúde e Estratégia de Saúde da Família. Gerência de Gestão da Atenção Primária à Saúde/ESF. Núcleo de Normas e Procedimentos. Manual de Atribuições dos Profissionais do Saúde da
Família. Apostila- versão preliminar. Brasília. 2009; 38 p.

17. Pinheiro FMC, Nóbrega-Therrien SM, Almeida MEL, Almeida MI. A Formação do Cirurgião-Dentista e a Promoção de Saúde no PSF. Rev. Odontol da UNESP. 2008; 37(1):6977.

18. Costa ICC, Fuscella MAP. Educação e Saúde: Importância da Integração dessas Práticas na Simplificação do Saber. Ação coletiva. 1999; 2(3):45-47.

19. Morita AC, Codato LAB, Higasi MS, Kasai MLHI. Visita Domiciliar: Oportunidade de Aprendizagem na Graduação em Odontologia. Rev. Odontol UNESP. 2010; 39(2):75-79.

20. Pretto SM, Franco F. Visitas Domiciliares como Estratégia de Educação em Saúde Relato de uma Experiência. Ação coletiva. 1999; 2(3):33-36.

21. Zanetti CHG. Saúde Bucal no Programa de Saúde da Família (PSF): proposição e programação. 2000. 28p.

22. Lima DC, Saliba NA, Moimaz SAS. Tratamento Restaurador Atraumático e sua utilização em saúde pública. RGO. 2008; 56(1):75-79.

23. Massoni ACLT, Pessoa CP, Oliveira AFB. Tratamento restaurador atraumático e sua aplicação na saúde pública. Rev. de Odontologia da UNESP. 2006; 35(3): 201-207.

24. Stelmann JP, Machado CR, Pirovane CL, Mello Pinto EC, Chevitarese L. T.R.A. no Programa de Saúde da Família. Rev. Rede de Cuidados em Saúde. 2008; 2(2):1-14.

25. Zanetti CHG, Machado MH. A utilidade como função para universalidade e equidade: 
uma análise formal da validade instrumental do ordenamento administrativo federal da assistência à saúde bucal no saúde da família. Rev. Brasileira de Medicina de Família e Comunidade. 2007; 2(8):312-13.

26. Brasil. Ministério da Saúde. Secretaria de Vigilância em Saúde. Guia de vigilância epidemiológica. $6^{\mathrm{a}}$ ed. Brasília: Ministério da Saúde, 2005.

27. Roncalli A, Unfer B, Costa ICC, Arcieri RM, Guimarães LOC, Saliba NA. Levantamentos Epidemiológicos em Saúde Bucal: Análise da Metodologia Proposta pela Organização Mundial da Saúde. Rev. Brasileira de Epidemiologia. 1998; 1(2):177-189.

28. Roncalli A, Frazão P, Pattussi MP, Araújo IC, Ely HC, Batista SM. Projeto SB2000: uma Perspectiva para a Consolidação da Epidemiologia em Saúde Bucal Coletiva. Rev. Bras. Odont. Saúde Coletiva. 2000; 1(2):9-25. 29. Pinto VG. Correções de Rumo para o Levantamento Epidemiológico em Saúde Bucal do ano 2000. Rev. Bras. Odont. Saúde Coletiva. 2000; 1(2):26-29.

30. Queiroz RCS, Portela MC, Vasconcellos MTL. Pesquisa sobre as Condições de Saúde Bucal da População Brasileira (SB Brasil 2003): seus dados não produzem estimativas populacionais, mas há possibilidade de correção. Cad Saúde Pública. 2009; 25:47-58.

31. Roncalli A. Projeto SB Brasil 2010: Elemento Estratégico na Construção de um Modelo de Vigilância em Saúde Bucal. Editorial do Cad. Saúde Pública. 2010; 26:428429.

32. Narvai PC, Antunes JLF, Moysés SJ, Frazão P, Peres MA, Peres KG, Sousa MLR, Roncalli
A. Validade Científica de Conhecimento Epidemiológico Gerado com Base no Estudo Saúde Bucal Brasil 2003. Cad. Saúde Pública. 2010; 26:647-670.

33. Spink MJP. O Conceito de Representação Social na Abordagem Psicossocial. Cad. Saúde Públ. 1993; 9(3):300-308.

34. Sevalho G. Uma Abordagem Histórica das Representações Sociais de Saúde e Doença. Cad. Saúde Públ. 1993; 9(3):349-363.

35. Gomes R, Mendonça EA, Pontes ML. As Representações Sociais e a Experiência da Doença. Cad. Saúde Pública. 2002; 18:120714.

36. Oliveira, MSBS. Representações Sociais e Sociedades: a Contribuição de Serge Moscovici. Rev. bras. Ci. Soc. 2004; 19(55):180-186. 